\title{
First analyses of the iOSG-type superconducting gravimeter at the low noise underground laboratory (LSBB URL) of Rustrel, France
}

\author{
Séverine Rosat ${ }^{1, \text { a }}$, Jacques Hinderer ${ }^{1}$, Jean-Paul Boy ${ }^{1}$, Frédéric Littel ${ }^{1}$, Daniel Boyer ${ }^{2}$, \\ Jean-Daniel Bernard ${ }^{1}$, Yves Rogister ${ }^{1}$, Anthony Mémin ${ }^{3}$, and Stéphane Gaffet ${ }^{2}$ \\ ${ }^{1}$ Institut de Physique du Globe de Strasbourg, UMR 7516, Université de Strasbourg/EOST, CNRS, \\ 5 rue Descartes, 67084 Strasbourg, France \\ ${ }^{2}$ LSBB URL, France \\ ${ }^{3}$ Université Côte d'Azur, CNRS, Observatoire de la Côte d'Azur, IRD, Géoazur, France
}

\begin{abstract}
In the last few years, the performance of the cryogenic gravity instruments has been further improved by the development of a new generation of superconducting gravimeter (SG): the so-called iOSG which is a superconducting gravimeter designed for observatory purpose with a heavier sphere than previous SGs. The first iOSG (iOSG-024) has been installed in July 2015 at the LSSB low background noise underground research laboratory in Rustrel (France), funded by the EQUIPEX MIGA (Matter wave-laser based Interferometer Gravitation Antenna) project and by the European FEDER 2006-2013 "PFM LSBB - Développement des qualités environnementales du LSBB". This instrument is operational since September 2015. We present the first tidal analyses of the 7-month time-varying gravity records of this newly installed instrument as well as the calibration results performed by parallel FG5 absolute gravity measurements. We also show the performances of iOSG-024 in terms of noise levels in the seismic (in the millihertz frequency range) band using a standardized procedure based on the computation of the residual power spectral densities over a quiet time period. The obtained noise levels are compared with other SG sites and with seismological reference noise models. The combination of the instrumental performance of the iOSG with the LSBB site properties makes this gravimetric station one of the quietest in the world, comparable to the lower sensor of the OSG-56 at BFO, at seismic frequencies.
\end{abstract}

\section{Introduction}

Superconducting Gravimeters (SGs) are relative instruments that continuously measure the time-varying gravity field at the Earth's surface. Since the development of the first commercialized SGs [1] in the 90s, the size and instrumental noise have been reduced [2]. In the last few years, the performance of the cryogenic gravity instruments has been further improved by the development of a new generation of superconducting gravimeter: the so-called iOSG which is a superconducting gravimeter designed for observatory purpose. A special SG prototype (called OSG-056), with sensor properties intermediate between the compact SG and the new iOSG, has already been recording since 2009 at the Black Forest

\footnotetext{
${ }^{a}$ Corresponding author: severine.rosat@unistra.fr
} 


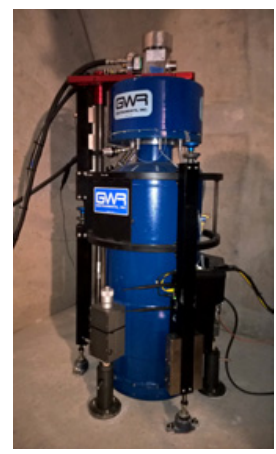

Figure 1. The iOSG-24 recording at the LSBB in Rustrel (France) since July 2015.

Observatory (BFO, Germany) with promising results in terms of sensitivity [2]. The OSG56 recording at $\mathrm{BFO}$ is indeed a dual-sphere instrument with a higher sphere weighting $4.3 \mathrm{~g}$ and a lower sphere which is heavier with a mass of $17.7 \mathrm{~g}$. So the lower sensor of OSG-56 (noted OSG-56_L) is similar to the iOSG. The first iOSG (iOSG-024; Fig. 1) has been installed at the LSSB low background noise underground research laboratory (URL) in Rustrel (France), funded by the EQUIPEX MIGA (Matter wave-laser based Interferometer Gravitation Antenna, [3]) project and was installed in July 2015. First data have been recorded since mid-September 2015.

The SG measurement relies on the magnetic feedback force of a levitated Niobium sphere in the vacuum. In order to keep the superconductivity of the sphere, the sensor is operating in a liquid Helium bath maintained at $4.2 \mathrm{~K}$. A liquefying system enables to recycle the Helium gas into liquid Helium making the human intervention for liquid Helium refilling unnecessary. A known source of anthropogenic noise is hence reduced.

The purpose of increasing the mass of the levitated sphere is to decrease the thermal noise due to Brownian motion inside the sensor. The spectral acceleration-noise power density $\left(P_{\text {thermal }}\right)$ due to Brownian motion in a simple mechanical oscillator is given by:

$$
P_{\text {thermal }}=4 k_{B} T \frac{\omega_{0}}{m Q}
$$

where $\omega_{0}$ is the natural frequency of the oscillator, $Q$ its quality factor and $m$ is the mass of the oscillating sphere [4]; $k_{B}$ is the Boltzmann constant and $T$ the temperature.

First, in Sect. 2, we show the calibration results. Next, in Sect. 3, we plot the noise levels at the seismic frequencies to compare the performance of the iOSG-24 with other worldwide SG stations. Finally, in Sect. 4, we present the noise levels in the tidal frequency bands.

\section{Calibration}

A usual way to calibrate relative gravimeters is to perform simultaneous absolute gravity measurements during a few days and to estimate a scale factor by least-squares adjustment of the tidal amplitudes (see e.g. [5]). This parallel FG5 measurement was realized on 5 days from October 8 to 12, 2015 with the FG5\#206 (serial number 206) absolute gravimeter owned by the Strasbourg gravimetric observatory. The resulting superposition of absolute gravity measurements and relative SG time-varying records is represented in Fig. 2. The obtained scale factor value is - $-451.2 \mathrm{~nm} / \mathrm{s}^{2} / \mathrm{V} \pm 0.27 \mathrm{~nm} / \mathrm{s}^{2} / \mathrm{V}$ (relative error of $0.06 \%$ ). 


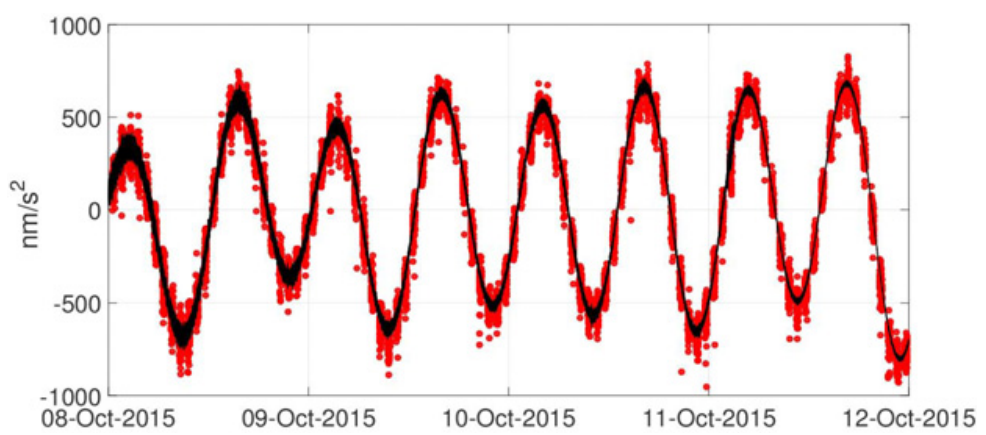

Figure 2. Superposition of the FG5\#206 absolute drop values (in red) with the iOSG-24 (in black) recording at the LSBB in Rustrel (France) after applying a scale factor of $-451 \mathrm{~nm} / \mathrm{s}^{2} / \mathrm{V}$.

\section{Seismic noise levels}

A standard procedure was introduced by $[6,7]$ to compare noise levels of various gravimetric sites. Since other worldwide SG data are available at a sampling interval of one minute, we down-sample the raw one-second iOSG-24 data to one minute using a low-pass filter with a cut-off period of two minutes. Then we consider daily time windows and remove solid tides for an elastic reference Earth model from the data as in [7]. A local atmospheric pressure reduction is also applied using a nominal admittance of $-3 \mathrm{~nm} / \mathrm{s}^{2} / \mathrm{hPa}$. The five quietest days in terms of RMS deviation are selected. Subsequently a Power Spectral Density (PSD) is computed using a smoothed periodogram estimate on these five quietest days.

The resulting PSD for the iOSG-24 is compared (Fig. 3) to some other noise levels at SG sites that were published in [2]. At frequencies larger than $1 \mathrm{mHz}$, the iOSG-24 has a noise level similar to the lower sensor of the BFO OSG-56 gravimeter which is among the lowest ones in the world. The OSG-56 is indeed a dual-sphere instrument where we denote by L the lower heavier sphere and by $\mathrm{U}$ the upper standard sphere. Below $1 \mathrm{mHz}$, the iOSG-24 has a noise level that becomes larger than BFO OSG-56_L. This could be due to the environment but further investigation is needed. Indeed, we cannot distinguish between environmental and instrumental noise with only one instrument at one site. When comparing the noise level of the lower sensor to the higher sensor of the BFO OSG-56, we see the efficiency of instrumental noise reduction by increasing the mass of the sphere (Eq. (1)) in the seismic frequency range considered here. However, it was shown in [2] that the noise reduction by increasing the mass of the sphere was not efficient any more below $0.1 \mathrm{mHz}$.

The high frequency decrease on Fig. 3 is due to the low-pass filtering that was applied before decimating data from one second to one minute.

A criterion of the quality of a site in terms of noise is also the observation of the incessant background free oscillations (the so-called "hum") [9], which are clearly visible on the iOSG24 data at LSBB (Fig. 4). We have plotted the quartiles of the Global Seismographic Network noise levels [10] that represent a more recent reference noise model than the New Low Noise Model NLNM [8]. At frequencies larger than $1 \mathrm{mHz}$, long-period seismometers have lower noise levels than the iOSG-24 (Fig. 4). Physical mechanisms to explain the incessant excitations of the fundamental seismic modes have been given by [11]. In fact, SGs are known to have a larger noise amplitude in the seismic band. This larger noise amplitude was partly indebted to the Brownian motion of the levitated sphere [12]. However, at frequencies smaller than $1 \mathrm{mHz}$, the $\mathrm{SG}$ has better performances due to a more efficient atmospheric pressure reduction [9]. 


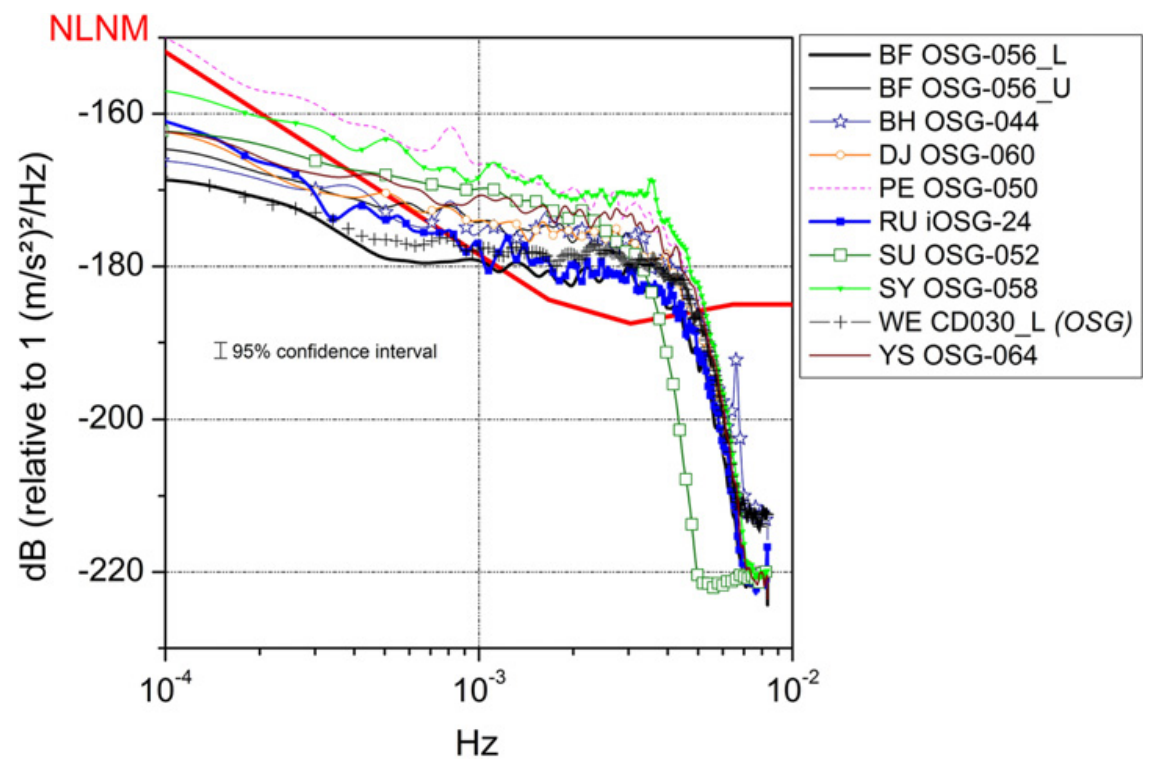

Figure 3. Noise level in the seismic band of the LSBB iOSG-24 site (named RU) compared with some worldwide SG sites: the dual-sphere OSG-56 at Black Forest Observatory (BF, lower -L- and upper -Usensor, Germany), the OSG-60 at Djougou (DJ, Benin), the OSG-44 at Bad-Homburg (BH, Germany), the OSG-50 at Pecny (PE, Czech Republic), the OSG-52 at Sutherland (SU, South-Africa), the OSG-58 at Syowa (SY, Antarctica), the lower-sphere of the dual-sensor CD030 at Wettzell (WE, Germany) and the OSG-64 at Yebes (YS, Spain). The seismological noise model (NLNM) [8] is represented by the thick red line. The color version of this figure is available only in the electronic edition.

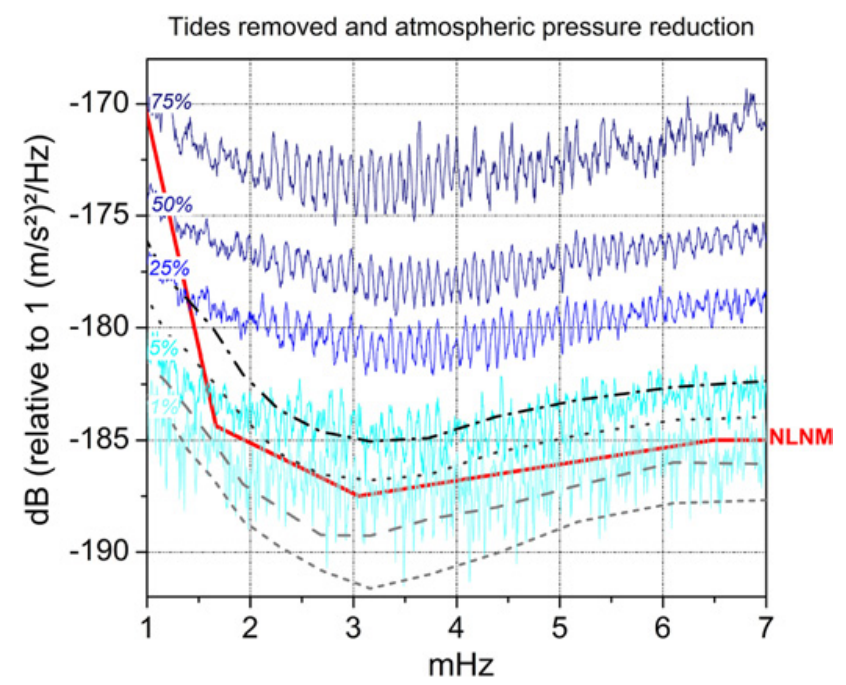

Figure 4. $1 \%, 5 \%, 25 \%, 50 \%$ and $75 \%$-tiles of daily Power Spectral Densities of the LSBB iOSG24 site (in cyan and blue lines) compared with the $1^{\text {st }}, 5^{\text {th }}, 25^{\text {th }}$ and $50^{\text {th }}$ percentiles of the Global Seismographic Network noise levels [10] respectively plotted in short-dashed gray, dashed gray, dotted gray and dot-dashed black lines. The seismological noise model (NLNM) [8] is represented by the thick red line. The color version of this figure is available only in the electronic edition. 

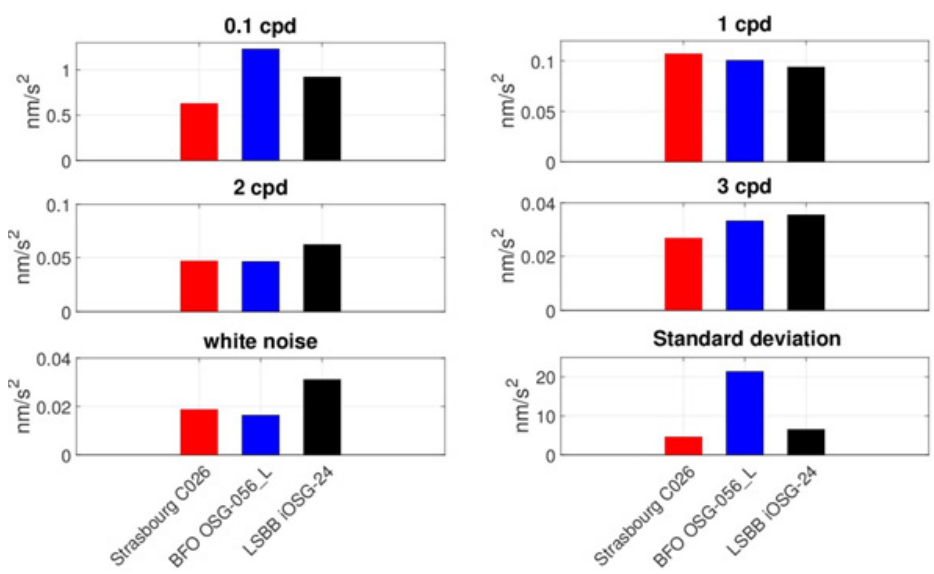

Figure 5. Average noise levels at frequency bands: $0.1,1,2$, and $3 \mathrm{cpd}$. The corresponding white noise amplitude as well as the standard deviations are also given. These average noise levels are obtained after an ETERNA tidal analysis on 212 days of data. The color version of this figure is available only in the electronic edition.

\section{Tidal analysis results}

The analysis of tidal waves in a gravimetric time series can be performed with the ETERNA software [13] which enables to retrieve a tidal model at the nanogal precision $(1 \mathrm{nGal}=$ $0.01 \mathrm{~nm} / \mathrm{s}^{2}$ ). An ETERNA tidal analysis performs a least-squares adjustment based on a tidal potential catalogue (we choose the Hartmann \& Wenzel catalogue [14]) to retrieve observed tidal amplitudes and phases, but also some noise levels in the tidal bands. These noise levels are the average FFT of gravity residuals from ETERNA analysis. They are normalized in order to be independent of the record lengths. The white noise estimate $\sigma$ is obtained from the mean power spectral density (PSD) of the gravity residuals after ETERNA processing through the relation PSD $=\sigma^{2} \mathrm{~T}_{0}$, where $\mathrm{T}_{0}$ is the sampling interval. The standard deviation of the gravity residual time series is also given.

The estimates of average noise levels in different frequency bands, 1, 2 and 3 cycle per day (cpd), are compared in Fig. 5 with the average noise levels obtained for the SG C026 recording at the gravimetric observatory of Strasbourg (France) and for the lower-sphere of the OSG-56 at BFO. We can see that the average noise level is slightly larger for the iOSG-24 at LSBB in the 2 and 3 cpd frequency bands than for the Strasbourg and BFO sites. Additional semi-diurnal and ter-diurnal tidal terms could be inserted in the least-squares adjustment in the tidal analysis in order to further improve the local tidal models and the removal of periodic terms at such frequencies. However, a longer time-series would be required, as we had only 212 days of records since the installation.

\section{Conclusion}

The environmental quiet site of the LSBB associated with the latest generation of superconducting gravimeters makes this gravimetric station one of the quietest in the world, particularly at seismic frequencies, with a noise level of $-180 \mathrm{~dB}$ at $1 \mathrm{mHz}$. The high-quality data it can provide, combined with global data, are very promising in the search for smallamplitude geophysical signals. 
The iOSG-24 gravimeter of the LSBB was funded by the EQUIPEX MIGA (Matter wave-laser based Interferometer Gravitation Antenna) ANR-11-EQPX-0028 and by the European FEDER 2006-2013 "PFM LSBB - Développement des qualités environnementales du LSBB".

\section{References}

[1] G. Goodkind, Phys. Rev., 84 (1999).

[2] S. Rosat, J. Hinderer, Bull. Seism. Soc. Am., 101, No. 3, 1233-1241 (2011).

[3] B. Canuel, L. Amand, A. Bertoldi, W. Chaibi, R. Geiger, J. Gillot, A. Landragin, M. Merzougui, I. Riou, S.P. Schmid and P. Bouyer, E3S Web of Conferences, 4 (2014).

[4] R.J. Warburton, H. Pillai, and R.C. Reineman, Int. Association of Geodesy (IAG) Symposium Proceedings (2010).

[5] J. Hinderer, D. Crossley, R.J. Warburton, Treatise on Geophysics, 3 (2007).

[6] D. Banka, Ph.D. Thesis, GDMB-Informationgesellschaft mbH, Clausthal, Germany (1997).

[7] D. Banka, D. Crossley, Geophys. J. Int., 139, 87-97 (1999).

[8] J. Peterson, U.S. Geol. Surv. Open-File Rept. 93-332, Albuquerque, New Mexico (1993).

[9] R. Widmer-Schnidrig, Bull. Seism. Soc. Am., 93, No. 3, pp. 1370-1380 (2003).

[10] J. Berger, P. Davis, G. Ekström, J. Geophys. Res., 109, B11307 (2004).

[11] K. Nishida, Geophys. J. Int., 199, 416-429 (2014).

[12] Richter B., Wenzel H.-G., Zürn W. and Klopping F., Phys. Earth Planet. Inter., 91, 131-148 (1995).

[13] H.-G. Wenzel, Bull. Inf. Marees Terr., 124, 9425-9439 (1996).

[14] T. Hartmann, H.-G. Wenzel, Geophys. Res. Lett., 22 (24), 3553-3556 (1995). 